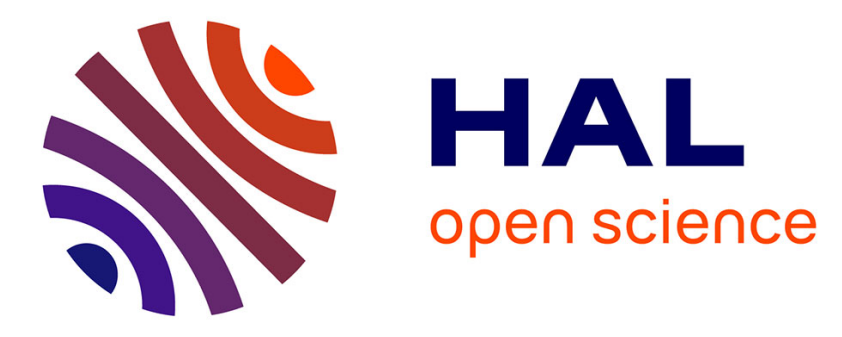

\title{
Cyber-Physical Design and Implementation of Distributed Event-Triggered Secondary Control in Islanded Microgrids
}

Yu Wang, Tung Lam Nguyen, Yan Xu, Zhengmao Li, Quoc-Tuan Tran, Raphael Caire

\section{To cite this version:}

Yu Wang, Tung Lam Nguyen, Yan Xu, Zhengmao Li, Quoc-Tuan Tran, et al.. Cyber-Physical Design and Implementation of Distributed Event-Triggered Secondary Control in Islanded Microgrids. IEEE Transactions on Industry Applications, 2019, 55 (6), pp.5631-5642. 10.1109/TIA.2019.2936179 . hal03213690

\section{HAL Id: hal-03213690 \\ https://hal.science/hal-03213690}

Submitted on 21 May 2021

HAL is a multi-disciplinary open access archive for the deposit and dissemination of scientific research documents, whether they are published or not. The documents may come from teaching and research institutions in France or abroad, or from public or private research centers.
L'archive ouverte pluridisciplinaire HAL, est destinée au dépôt et à la diffusion de documents scientifiques de niveau recherche, publiés ou non, émanant des établissements d'enseignement et de recherche français ou étrangers, des laboratoires publics ou privés. 


\title{
Cyber-Physical Design and Implementation of Distributed Event-Triggered Secondary Control in Islanded Microgrids
}

\author{
Yu Wang, Member, IEEE, Tung Lam Nguyen, Student Member, Yan Xu, Senior Member, IEEE, Zhengmao Li, Student \\ Member, IEEE, Quoc-Tuan Tran, Senior Member, IEEE, Raphael Caire, Member, IEEE.
}

\begin{abstract}
Microgrid (MG) is a cyber-physical system with coupled power and communication networks. The centralized secondary control of MGs with periodical communications restricts system efficiency and resilience. This paper proposes a distributed event-triggered secondary control scheme in islanded MGs with its cyber-physical implementation. The proposed control scheme operates with reduced frequency of communications depending on the MG states change 'events' (e.g. load variations and communication failures). Besides, the secondary control objectives, including frequency/voltage regulation and accurate real/reactive power sharing, are decoupled into two timescales. Instead of designing eventtriggering conditions (ETCs) for each secondary control functions, only ETCs for power sharing control in slower timescale are designed. Thus, the communication burden is significantly reduced since communications among neighbour controllers are only needed at the event-triggered time. The proposed controller has been tested on a hardware-in-the-loop (HIL) platform, where the physical system is modelled in OPAL-RT and the cyber system is realized in Raspberry Pis. The control effectiveness is validated by the HIL results.
\end{abstract}

Index Terms - distributed event-triggered control, cyberphysical systems, microgrids, hardware-in-the-loop, raspberry pi.

\section{INTRODUCTION}

\section{A. Background}

Microgrids (MGs), as small-scale power systems, are electrically integrated with various distributed generators (DGs) and energy storage systems, and loads [1]-[3]. In addition, the communication system also plays a vital role in monitoring and control the MG. Therefore, the MG is a typical cyber-physical system with interacted electrical and communication networks [4]. In cyber-physical system research, the networked control systems and distributed consensus algorithms have received significant research interests for designing the communication and control architecture of the MGs [5]-[7].

As for the control of MGs, the hierarchical control is a standardized framework [8]-[10], including primary, secondary, and tertiary control levels. The droop based primary control is for autonomous power sharing with only local measurements [8]. The main objectives of secondary control are to restore the voltage/frequency to the nominal values and additionally achieving accurate power sharing, harmonics sharing, unbalanced voltage compensation [11]-[13]. The tertiary

Y. Wang, Y. Xu and Z. Li are with Nanyang Technological University, Singapore, Nanyang 50 Ave, 639798.

T. L. Nguyen, and R. Caire are with University Grenoble Alpes, G2Elab, F38000 Grenoble, France. control usually refers to the economic dispatch and optimal power flow for MGs [14].

\section{B. Literature Survey}

This paper focuses on the secondary control of MGs, which are typically achieved in two ways, i.e. centralized control and distributed control. The centralized secondary control or automatic generation control are widely used in existing power systems and MG systems [15], [16]. However, the central controller suffers extensive computation and communication burdens when the system scale is large, and it is inherently vulnerable to communication and single-point failures. As alternative approaches to overcome the above limitations, the distributed control algorithms via only peer-to-peer communications are widely investigated [11]-[13], [17]-[19]. The frequency restoration and accurate real power sharing processes can be achieved at the same time, as the system frequency is global synchronized information in steady-state [11], [13], [17]. Since the line impedance of the MG network, bus voltages are local variables with different values, the precise voltage restoration and reactive power sharing cannot be concurrently achieved [13], [17]. Various distributed secondary controllers of MGs are investigated, such as linear control [13], [17] optimal control [18], finite-time control [11], [19] etc. However, in the above literature, the control and communication are conducted on a periodic and frequent basis, which is not computationally efficient in most cases. In fact, the secondary controllers are not necessary to be periodically and frequently operated. Rather, they can be triggered depending on the state change events in MGs.

In such circumstances, the event-triggered approaches are proposed to reduce the communication and computation burden under the distributed control framework [20], [21]. The eventtriggered controllers are not performed at predefined instants but only when required. The controllers are only updated when a local measurement error exceeds a tolerable bound, thereby significantly reducing communication burden while maintaining a satisfactory control performance [21]. So far, several research works have reported the distributed eventtriggered secondary control of droop based DGs in islanded MGs in [22]-[24]. For each secondary control function, one specific event-triggered controller and its triggering condition are designed [22]-[24]. In practical implementation, all the

Q. T. Tran is with Alternative Energies and Atomic Energy Commission (CEA), National Institute for Solar Energy (INES), F-73375 Le Bourget-duLac, France.

Corresponding author: Yan Xu. (email: xuyan@ntu.edu.sg) 
event-triggered secondary controllers should share only one common communication network. The more event-triggered controllers are considered, the more communication burdens are caused. This paper aims to address this ignored but practical problem, where the control of $\omega / P$ and $V / Q$ are decoupled in different timescales and the event-triggered condition for only slow dynamics are designed. Besides, most research works for the event-triggered secondary control of MGs only conducted simulation studies [22]-[24]. As distributed event-triggered control is inherently dependent on communications, the validation of the designed controllers on a realistic environment becomes quite important. Therefore, a multi-agent system (MAS) is built up by multiple Raspberry Pis (R-Pis) to investigate the proposed control methods [25], [26].

\section{Contributions}

In this paper, a distributed, event-triggered, and time decoupled secondary control is proposed for frequency/voltage restoration and accurate power sharing in islanded cyberphysical MGs. Towards practical applications, hardware-inthe-loop (HIL) test is conducted to validate the proposed method in a more realistic environment. Significant extensions and improvement have been made based on our previous work in [27]. The novelties of this paper are as follows:

1) This paper proposes a new distributed event-triggered secondary control scheme for islanded MGs. The secondary control problems are decoupled into two timescales and eventtriggering conditions (ETCs) are designed to significantly reduce the communication burdens in the cyber system.

2) The design and implementation of MGs from the perspective of the cyber-physical system are presented. The physical system of MG is emulated in real-time by OPAL-RT. The cyber system of MG is realized in a MAS, which consists of multiple R-Pis installed with google remote process call (gRPC). The platform could emulate the MG governed by MAS more realistically.

3) The proposed distributed event-trigged control is validated on the cyber-physical MG testbed. The hardware-inthe-loop (HIL) results demonstrate the effectiveness of the proposed method under the events in both the physical system (load variations) and the cyber system (communication failures).
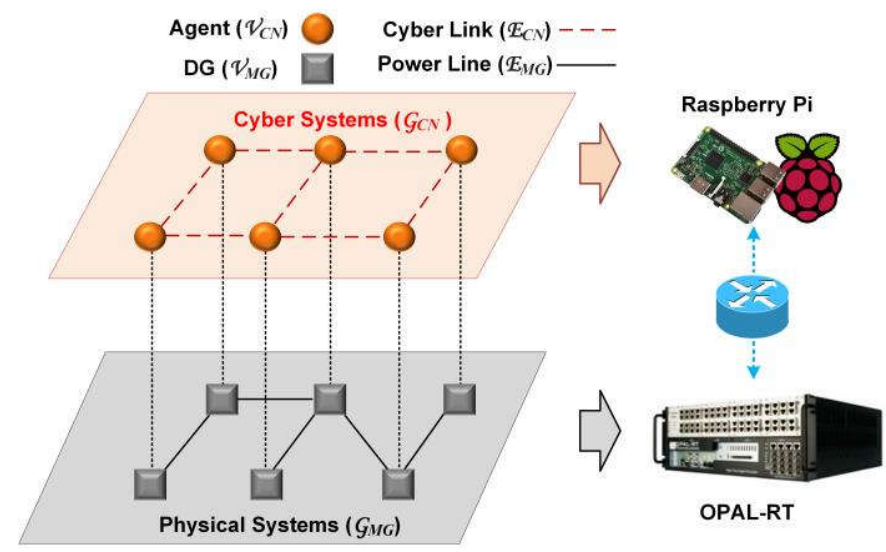

Fig. 1. A cyber-physical MG testbed.

\section{CYBER-PhysicAl MODELling OF MiCROGRIDS}

In this paper, the proposed distributed event-triggered control is designed and implemented from a cyber-physical system perspective. A cyber-physical MG testbed is built up with real-time simulator OPAL-RT and R-Pi cluster based HIL platform, as shown in Fig. 1. The electric network of MG and its local controllers, as the physical system, are simulated in OPAL-RT. The MAS and its communication network, as the cyber system, are realized by a cluster of R-Pis. Each R-Pi hosts an agent which is programmed to communicate with other agents and calculate the proposed event-triggered algorithm.

\section{A. Physical System}

1) Electric Networks of MGs. Consider a MG with $N$ DGs (indexed by $i=1,2, \ldots, N$.). The MGs can be represented by a complex-weighted graph $G_{M G}=\left(\mathcal{V}_{M G}, \mathcal{E}_{M G}\right)$, where the nodes $\mathcal{V}_{M G}$ represent the buses with DGs, and the edges $\mathscr{E}_{M G} \subseteq \mathcal{V}_{M G} \times$ $V_{M G}$ represent the line connections, as demonstrated in Fig. 1. The topology and line impedance of the MG network are depicted by an adjacency matrix $Z=\left[Z_{i k}\right] \subseteq \mathrm{C}$. If there is a line connection between $i$ th and $k$ th DGs, $Z_{i k}=R_{i k}+j X_{i k}$, where $R_{i k}+j X_{i k}$ are resistance and reactance, otherwise $Z_{i k}=0$. Since the output impedance of DGs are inductive, the active and reactive power injections $P_{i}$ and $Q_{i}$ of DG $i$ are represented by [17]

$$
\begin{gathered}
P_{i}=\sum_{j=1}^{N} \frac{V_{i} V_{j}}{X_{i j}} \sin \left(\theta_{i}-\theta_{j}\right)+P_{i}^{L} \\
Q_{i}=\frac{V_{i}^{2}}{X_{i j}}-\sum_{j=1}^{N} \frac{V_{i} V_{j}}{X_{i j}} \cos \left(\theta_{i}-\theta_{j}\right)+Q_{i}^{L}
\end{gathered}
$$

where $V_{i}$ and $\theta_{i}$ are bus voltage and angle of bus $i, P_{i}^{L}$ and $Q_{i}^{L}$ are real and reactive power of the load at bus $i$.

2) Primary Control. In a single $\mathrm{MG}$, the difference of $\theta_{i k}=\theta_{i}-\theta_{k}$ is small, which means that $\sin \left(\theta_{i}-\theta_{k}\right) \approx \theta_{i}-\theta_{k}$, $\cos \left(\theta_{i}-\theta_{k}\right) \approx 1$ in (1) and (2). Thus the real power of DG $i$ can be controlled by the difference of phase angle, while the reactive power of DG $i$ can be controlled by the difference of voltage magnitude. It comes out the basic mechanism of droop control (i.e., $P$ versus $\omega$ and $Q$ versus $V$ ) as follows:

$$
\begin{aligned}
& \omega_{i}=\omega_{i}^{\text {nom }}-K_{i}^{P} P_{i} \\
& V_{i}=V_{i}^{\text {nom }}-K_{i}^{Q} Q_{i}
\end{aligned}
$$

where $\omega_{i}^{\text {nom }}$ and $V_{i}^{\text {nom }}$ are the nominal set-points of primary droop control. $K_{i}^{P}$ and $K_{i}^{Q}$ are droop coefficients of real and reactive power, which are typically chosen by the power rating of the corresponding DG [8]. The inner voltage and current control loops also belong to the primary control, which have a much faster response than that of droop control. Therefore, the DG unit can be modelled as a controlled voltage source with an output impedance, which is governed by droop control as well as secondary control [17].

Remark 1: In this paper, the dispatchable DG units are operated under the proposed secondary control scheme. The renewable energy resources (RESs) which operates in maximum power tracking mode are viewed as non-dispatchable units. The resistive loads (RLs) are directly connected in the 


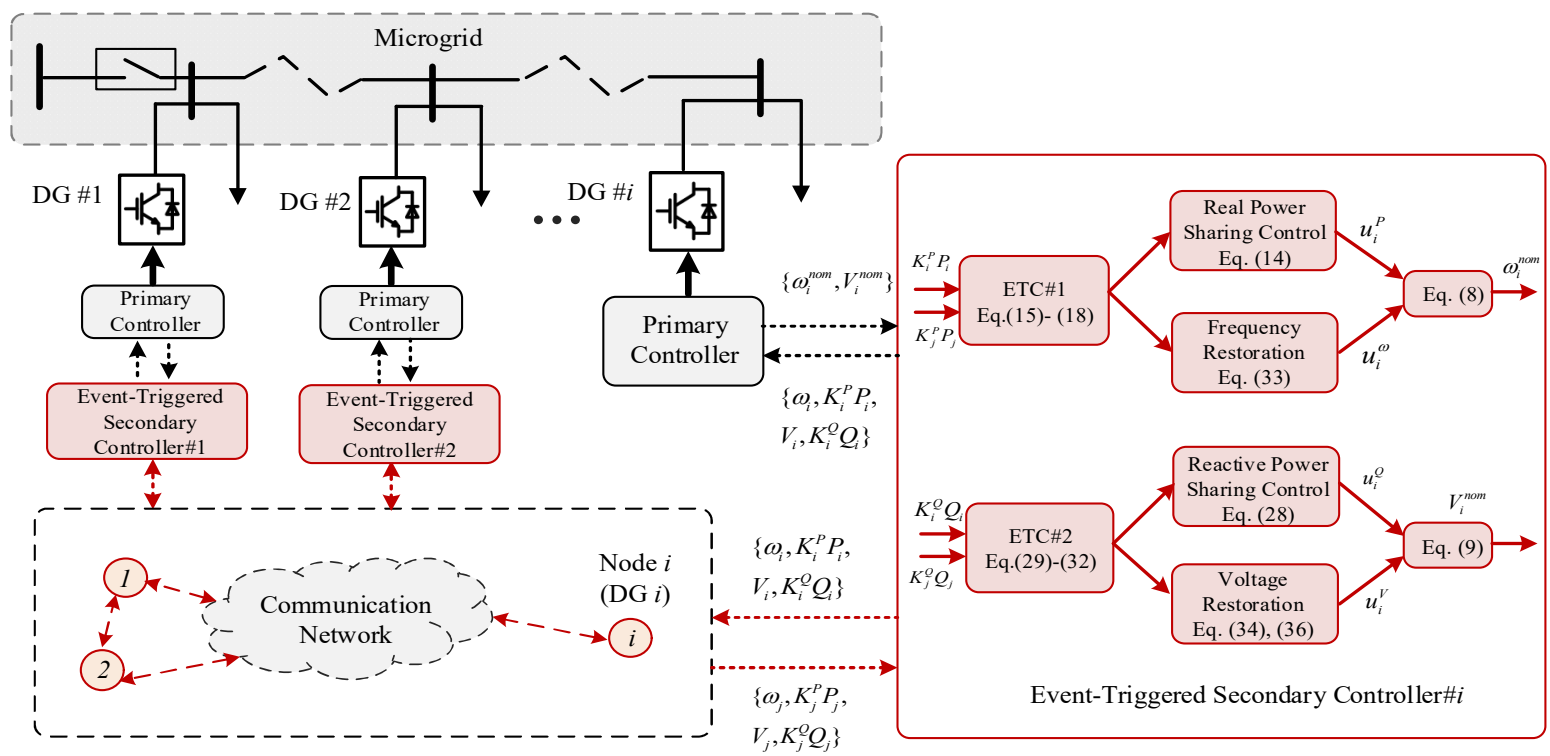

Fig. 2. The framework of proposed distributed event-triggered secondary control.

MG without any interfacing devices. Motor drives and power electronic interfaced loads are modeled as constant power loads (CPLs) as their tightly-regulated consumed power. The RESs, CPLs, and RLs can be combined as the lumped load $P_{L}=P_{C P L}+P_{C P L}-P_{R E S}$, which is widely adopted in current research of secondary control in MGs [28]. It should be noted that the proposed method is aimed to solve the power sharing problem at the fundamental frequency. The non-linear loads which lead to harmonics are not further considered but have been discussed in [29].

\section{B. Cyber System}

1) Communication Networks of MGs. The communication network of a MG with $N$ DGs is depicted by a graph: $G_{C N}=(\mathcal{V}$ $\left.{ }_{C N}, E_{C N}\right)$ with a set of nodes $\mathcal{V}_{C N}$ and a set of edges $\mathbb{E}_{C N}=\mathcal{V}_{C N} \times$ $\mathcal{V}_{C N}$, as shown in Fig.1. The nodes in graph $G_{C N}$ (communication agents) are one to one corresponding to nodes in graph $G M G$ $(D G s)$. The edges in $G_{C N}$, which represent communication links for data exchange, can be different from the electrical network in $G_{M G}$. The adjacency matrix of $G_{C N}$ is defined as $\mathcal{A}=\left[a_{i j}\right]$. The element $a_{i j}$ represents the information exchanged between agents $i$ and $j$, where $a_{i j}=1$ if agents $i$ and $j$ are connected, otherwise, $a_{i j}=0$. The in-degree matrix is defined as $\mathcal{D}=\operatorname{diag}\left[d_{i}\right]$, where $d_{i}=\sum_{i=1}^{N} a_{i j}$. The Laplacian matrix is defined as $\mathcal{L}=\left[l_{i j}\right]$, and $\mathcal{L}=\mathscr{D}-\mathcal{A}$. The convergence rate is determined by the minimum nonzero eigenvalue of $\mathcal{L}$, which is called algebraic connectivity [30].

2) Event-Triggered Mechanism. In the event-triggered control and communication scheme, the data of agent $i$ is transmitted to neighbour agents only when the ETC is satisfied. The 'event' refers to any disturbance which alters the system states. The following two notations are introduced [31]:

Triggered time sequence: $\left\{t_{k} \mid k=1,2, \ldots\right\} \cdot t_{k}$ denotes the time instant of the $k$ th triggering event, where each agent broadcasts its data to neighbours. The transmitted data is assumed to be successfully stored by a zero-order-holder.
State measurement error: $e(t)=x\left(t_{k}\right)-x(t), t \in\left[t_{k}, t_{k+1}\right)$. $x(t)$ is the current system state variable, while $x\left(t_{k}\right)$ is the system state variable of the $k$ th triggering event.

In centralized event-triggered mechanism, all agents are triggered by one ETC, the next time instant $t_{k+1}$ can be calculated by

$$
t_{k+1}=t_{k}+\min _{l}\left\{l \mid e^{T}(t) \Phi e(t) \geq \sigma x^{T}\left(t_{k}\right) \Phi x\left(t_{k}\right)\right\}
$$

where $\Phi$ and $\sigma$ are matrix and constant to be designed.

It is noted that this triggering mechanism relies on a central controller to update the triggered time sequence for each agent. As only peer-to-peer communications are considered, a fully distributed secondary event-trigger control will be introduced in the cyber system in the next section.

\section{Distributed EVEnT-TRIGGered SeCONDARY CONTROL}

The general framework of the proposed control scheme is shown in Fig. 2. The secondary controller of a DG processes the distributed event-triggered and exchange data with local primary controller and neighbouring DGs. When either of ETCs is satisfied, the secondary controller will update control signals to the primary control. In the meantime, the data packets of MG state variables at this event-triggered time will be sent to the neighboring DG controllers. The detailed mechanism for each part will be elaborated in the following subsections.

\section{A. Problem Formation and Control Objectives}

Problem Formation: In order to formulate the problem of MG secondary control, differentiating (1) and (2) yields

$$
\begin{aligned}
& \dot{\omega}_{i}=\dot{\omega}_{i}^{\text {mom }}-K_{i}^{P} \dot{P}_{i} \\
& \dot{V}_{i}=\dot{V}_{i}^{\text {nom }}-K_{i}^{Q} \dot{Q}_{i}
\end{aligned}
$$

Based on the feedback linearization, the secondary controller for frequency and voltage restoration can be formulated as, $\dot{\omega}_{i}=u_{i}^{\omega}, \dot{V}_{i}=u_{i}^{V}$. In the meantime, frequency and voltage can be considered in steady-state when performing the accurate power sharing control, one can obtain that $K_{i}^{P} \dot{P}_{i}=u_{i}^{P}$, 
$K_{i}^{Q} \dot{Q}_{i}=u_{i}^{Q}[19],[24]$.

From (6) and (7), the nominal set-points $\omega_{i}^{\text {nom }}$ and $V_{i}^{\text {nom }}$ in droop control can be obtained from:

$$
\begin{aligned}
\omega^{\text {nom }} & =\int\left(\dot{\omega}_{i}+K_{i}^{P} \dot{P}_{i}\right) d t=\int\left(u_{i}^{\omega}+u_{i}^{P}\right) d t \\
V^{n o m} & =\int\left(\dot{V}_{i}+K_{i}^{P} \dot{Q}_{i}\right) d t=\int\left(u_{i}^{V}+u_{i}^{Q}\right) d t
\end{aligned}
$$

where the $\omega^{\text {nom }}$ is controlled by $u_{i}^{\omega}$ and $u_{i}^{P}$, while $V^{\text {nom }}$ is controlled by $u_{i}^{\omega}$ and $u_{i}^{P}$.

Control Objectives: The control objectives of the proposed distributed event-triggered secondary control are as follows:

1. Accurate real power sharing of DGs and frequency restoration of MGs, i.e.

$$
\begin{array}{r}
\lim _{t \rightarrow \infty}\left|K_{i}^{P} P_{i}(t)-K_{i}^{P} P_{j}(t)\right|=0, \forall i \neq j \\
\lim _{t \rightarrow \infty}\left|\omega_{i}(t)-\omega^{r e f}\right|=0, \forall i \in G
\end{array}
$$

2. Accurate reactive power sharing of DGs and averaged voltage restoration of MGs, i.e.

$$
\begin{gathered}
\lim _{t \rightarrow \infty}\left|K_{i}^{Q} Q_{i}(t)-K_{i}^{Q} Q_{j}(t)\right|=0, \forall i \neq j \\
\lim _{t \rightarrow \infty}\left|\frac{1}{N} \sum_{i=1}^{N} V_{i}(t)-V^{r e f}\right|=0,
\end{gathered}
$$

The first objective will be achieved by control inputs $u_{i}^{\omega}\left(t_{k}^{P i}\right)$ and $u_{i}^{P}\left(t_{k}^{P_{i}}\right)$ with the triggered time sequence $\left\{t_{k}^{P_{i}} \mid k=1,2, \ldots\right\}$ for each agent. The second objective will be achieved by control inputs $u_{i}^{V}\left(t_{k}^{O i}\right)$ and $u_{i}^{Q}\left(t_{k}^{O i}\right)$ with the triggered time sequence $\left\{t_{k}^{Q_{i}} \mid k=1,2, \ldots\right\}$ for each agent.

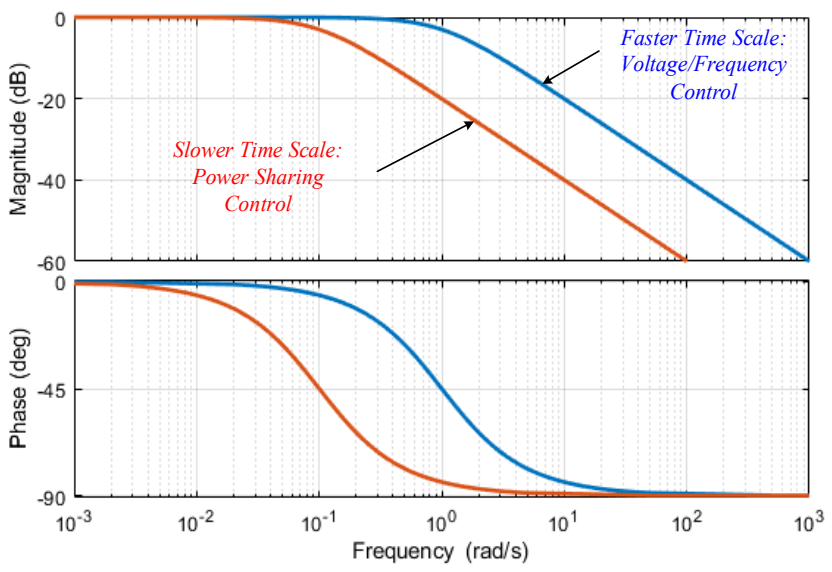

Fig. 3. Timescale decoupled secondary control: frequency-domain response.

Remark 2: In this paper, the secondary control of $\omega / P$ and $V / Q$ are decoupled into two timescales. Fig. 3 is used to illustrate the frequency-domain response of the timescale decoupling. The frequency/voltage control can be realized with a faster response speed than power sharing control, i.e. the stabilization time holds $T^{P} \approx 10 T^{w}$ and $T^{Q} \approx 10 T^{V}$.

There are mainly two advantages of the timescale decoupling in secondary control. 1) For each secondary control functionalities, the controllers can be separately designed for desired performance. 2) As all the secondary controllers share one common communication network, designing of four separate ETCs will increase the communication burden. Therefore, it is proposed to design only two ETCs based on the states of $P$ and $Q$ in a slower timescale. This design avoids the overlap of triggering conditions in different timescales, which further reduces the communication burden in the cyber system.

\section{B. Controller Design}

In this section, the distributed event-triggered secondary controllers for $P, \omega, Q$ and $V$ with two ETCs are presented.

1) Real Power Sharing Control. The power sharing control among each DG owns the dynamics of a first-order system $K_{i}^{P} \dot{P}_{i}=u_{i}^{P}$. Accurate power sharing can be achieved by the following control laws:

$$
u_{i}^{P}(t)=k_{i}^{P} \delta_{i}^{P}(t)
$$

where the control gain $k_{i}^{P}>0$, and $\delta_{i}^{P}(t)$ is defined as

$$
\delta_{i}^{P}(t)=\sum_{j=1}^{N} a_{i j}\left[K_{j}^{P} P_{j}\left(t_{k}^{P j}\right)-K_{i}^{P} P_{i}\left(t_{k}^{P i}\right)\right]
$$

Note that the control law in (14) and (15) is updated only at the event-triggered time rather than fixed periodical time interval.

The state measurement error is calculated from

$$
e_{i}^{P}(t)=K_{i}^{P} P_{i}\left(t_{k}^{P i}\right)-K_{i}^{P} P_{i}(t), t \in\left[t_{k}^{P i}, t_{k+1}^{P i}\right)
$$

ETC\#1: Considering a connected graph $G_{C N}$ and dynamics of real power sharing as $K_{i}^{P} \dot{P}_{i}=u_{i}^{P}$, the control law in (14) and (15) can reach consensus condition in (10), if the eventtriggered time sequence is generated as follows:

$$
t_{k}^{P i}=\inf \left\{t>t_{k-1}^{P i} \mid f_{i}^{P}(t)=0\right\}
$$

where the triggering function $f_{i}^{P}(t)$ of agent $i$

$$
f_{i}^{P}(t)=\left\|e_{i}^{P}(t)\right\|^{2}-\frac{\sigma_{i}^{P} \alpha_{i}^{P}\left(1-\alpha_{i}^{P} d_{i}\right)}{d_{i}}\left\|\delta_{i}^{P}(t)\right\|^{2}
$$

The coefficients $0<\sigma_{i}^{P}<1,0<\alpha_{i}^{P}<1 / d_{i} ; d_{i}=\sum_{i=1}^{N} a_{i j}$ is the element in the in-degree matrix $\mathscr{D}$.

Stability Proof: For simplicity of the proof, we omit the superscript $P$, abbreviate $x(t)$ to $x$ and denote $p_{i}=K_{i}^{P} P_{i}$, $x=\left[x_{1}, x_{2}, \ldots, x_{N}\right]^{T}$. The closed loop system can be written as

$$
\dot{p}=-k \mathcal{L}(p+e)
$$

Selecting the Lyapunov function candidate as:

$$
V=\frac{1}{2} p^{T} \mathcal{L} p
$$

Then taking derivative of (20), it can be derived:

$$
\dot{V}=\frac{1}{2} p^{T} \mathcal{L} \dot{p}=-k p^{T} \mathcal{L}(\mathcal{L} p+\mathcal{L} e)=-k \delta^{T} \delta-k \delta^{T} \mathcal{L} e
$$

Expanding the above equation (21):

$$
\begin{aligned}
& \dot{V}=-k_{i} \sum_{i=1}^{N} \delta_{i}^{2}-k_{i} \sum_{i=1}^{N} \sum_{j \in N_{i}} \delta_{i}\left(e_{i}-e_{j}\right) \\
& =-k_{i} \sum_{i=1}^{N} d_{i} \delta_{i} e_{i}-k_{i} \sum_{i=1}^{N} \delta_{i}^{2}+k_{i} \sum_{i=1}^{N} \sum_{j \in N_{i}} \delta_{i} e_{j}
\end{aligned}
$$

Using the inequality

$$
|x y| \leq \frac{\alpha}{2} x^{2}+\frac{1}{2 \alpha} y^{2}, \alpha>0
$$

We can obtain the upper bound of $\dot{V}$ as follows:

$$
\dot{V} \leq-k_{i} \sum_{i=1}^{N} \delta_{i}^{2}+k_{i} \sum_{i=1}^{N} \alpha d_{i} \delta_{i}^{2}+k_{i} \sum_{i=1}^{N} \frac{1}{2 \alpha} d_{i} e_{i}^{2}+k_{i} \sum_{i=1}^{N} \sum_{j \in N_{i}} \frac{1}{2 \alpha} e_{j}^{2}
$$


Since the graph is symmetric, by interchanging the indices of the last term we get

$$
\dot{V} \leq-k_{i} \sum_{i=1}^{N}\left(1-\alpha d_{i}\right) \delta_{i}^{2}+k_{i} \sum_{i=1}^{N} \frac{1}{\alpha} d_{i} e_{i}^{2}
$$

Assume that $\alpha$ satisfies $0<\alpha<1 / d_{i}$. Then, if the following condition is met

$$
e_{i}^{2} \leq \frac{\sigma_{i} \alpha_{i}\left(1-\alpha_{i} d_{i}\right)}{d_{i}} \delta_{i}^{2}
$$

It can be further derived that

$$
\dot{V} \leq k_{i} \sum_{i=1}^{N}\left(\sigma_{i}-1\right)\left(1-\alpha d_{i}\right) \delta_{i}^{2} \leq 0
$$

which is negative definite for $0<\sigma_{i}<1$.

Therefore, the stability of the closed-loop system is proved. The minimal inter-event times are lower bounded away from zero as discussed in [20], which are not illustrated here as space limits.

Remark 3: Fig. 4 is used to illustrate the working principle of ETC\#1. ETC\#1 actually compares the values of two terms $\left\|e_{i}^{P}(t)\right\|^{2}$ and $\frac{\sigma_{i}^{P} \alpha_{i}^{P}\left(1-\alpha_{i}^{P} d_{i}\right)}{d_{i}}\left\|\delta_{i}^{P}(t)\right\|^{2}$ in (18). The blue curve refers to the term of $\left\|e_{i}^{p}(t)\right\|^{2}$, which will keep increasing until the next event-triggered time. The upper boundary is given by the red curve, which will keep decreasing as the control eliminates the error among agents $\left\|\delta_{i}^{P}(t)\right\|$. Once the condition is satisfied, $\left\|e_{i}^{P}(t)\right\|^{2}$ will be updated to zero with the state $K_{i}^{P} P_{i}\left(t_{k}^{P i}\right)$ equalling to the current state $K_{i}^{P} P_{i}(t)$. During the intervals between two time instants, no communication is required. The ETC of each agent is only based on the information of its own and neighbour agents, so a fully distributed event-triggered control scheme is developed. In practical application, the triggering condition can be terminated when $\left\|e_{i}^{P}(t)\right\|$ is smaller than a certain value.

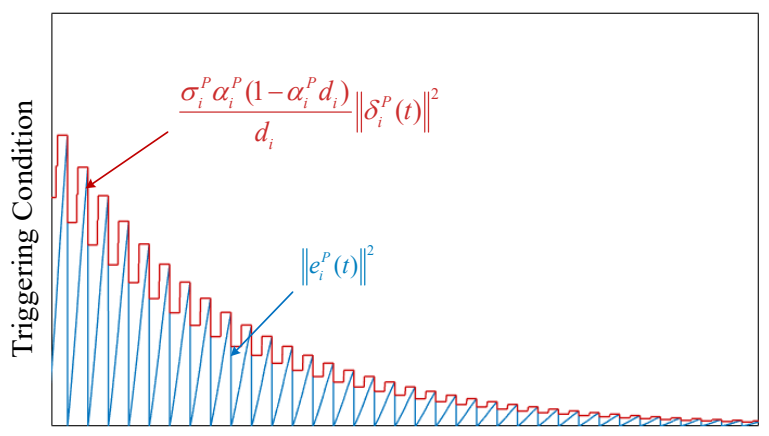

Time

Fig. 4. Illustration of the event-triggering condition.

2) Reactive Power Sharing Control: Aligning with the ideas of timescale decoupling, the event-triggered control of reactive power sharing and its ETC are designed. The reactive power sharing among each DG owns the dynamics of a first-order system $K_{i}^{Q} \dot{Q}_{i}=u_{i}^{Q}$. The accurate reactive power sharing can be achieved by the following control laws:

$$
u_{i}^{Q}=k_{i}^{Q} \delta_{i}^{Q}(t)
$$

where the control gain $k_{i}^{Q}>0$ and $\delta_{i}^{Q}(t)$ is defined as

$$
\delta_{i}^{Q}(t)=\sum_{j=1}^{N} a_{i j}\left[K_{j}^{Q} Q_{j}\left(t_{k}^{Q j}\right)-K_{i}^{Q} Q_{i}\left(t_{k}^{Q i}\right)\right]
$$

The state measurement error is given as

$$
e_{i}^{Q}(t)=K_{i}^{Q} Q_{i}\left(t_{k}^{Q i}\right)-K_{i}^{Q} Q_{i}(t), t \in\left[t_{k}^{Q i}, t_{k+1}^{Q i}\right)
$$

ETC\#2: Considering a connected graph $G_{C N}$ and dynamics of reactive power sharing as $K_{i}^{Q} \dot{Q}_{i}=u_{i}^{Q}$, the control law in (28) and (29) can reach consensus condition in (12), if the eventtriggered time sequence is generated as follows:

$$
t_{k}^{Q i}=\inf \left\{t>t_{k-1}^{Q i} \mid f_{i}^{Q}(t)=0\right\}
$$

where the triggering function $f_{i}^{Q}(t)$ of agent $i$

$$
f_{i}^{Q}(t)=\left\|e_{i}^{Q}(t)\right\|^{2}-\frac{\sigma_{i}^{Q} \alpha_{i}^{Q}\left(1-\alpha_{i}^{Q} d_{i}\right)}{d_{i}}\left\|\delta_{i}^{Q}(t)\right\|^{2}
$$

The coefficients $0<\sigma_{i}^{Q}<1,0<\alpha_{i}^{Q}<1 / d_{i} ; d_{i}=\sum_{i=1}^{N} a_{i j}$ is the element in in-degree matrix $\mathscr{D}$.

The stability proof has a similar process of ETC\#1, which is not discussed here for simplicity.

3) Frequency Control: As discussed above, the frequency control has faster dynamics of real power sharing control, thus the system state $\omega$ can reach steady-state faster than $P$. Instead of designing two separate ETCs for $\omega$ and $P$, frequency control can also be triggered by ETC\#1. The distributed frequency controller can be designed as

$$
u_{i}^{\omega}=k_{i}^{\omega}\left\{\sum_{j=1}^{N} a_{i j}\left[\omega_{j}(t)-\omega_{i}(t)\right]+\left[\omega^{r e f}-\omega_{i}(t)\right]\right\}
$$

where $k_{i}^{\omega}>0$ and $t \in\left\{t_{k}^{P i} \mid k=1,2, \ldots\right\}$.

4) Voltage Control: The voltage control and the reactive power control share the ETC\#2. In this paper, the weighted average bus voltage is estimated and restored, as the trade-off between accurate reactive power sharing and voltage regulation Firstly, the average voltage value $\bar{V}_{i}$ of each DG is estimated by a distributed dynamic consensus algorithm. The estimator of DG $i$ updates its own output $\bar{V}_{i}$ by processing the neighbours' estimation $\bar{V}_{j}$, and calculates the local voltage measurement $V_{i}$ as follows:

$$
\dot{\bar{V}}_{i}(t)=\dot{V}_{i}(t)+\sum_{j=1}^{N} a_{i j}\left[\bar{V}_{j}(t)-\bar{V}_{i}(t)\right]
$$

where (34) can be represented in matrix form as $\dot{\bar{V}}(t)=\dot{V}(t)-L \bar{V}(t)$, and the transferred into the frequency domain as $\bar{V}(s)=s\left(s I_{N}+L\right)^{-1} V(s)$. For an undirected and connected $G_{C N}$, the system is stable [32]. The estimator can steer each DG's voltage observations asymptotically converge to the weighted average value of all DGs' actual voltage magnitudes. Using the final value theorem, it gives

$$
\lim _{t \rightarrow \infty} \bar{V}_{i}(t)=\lim _{t \rightarrow \infty} \frac{1}{N} \sum_{i=1}^{N} V_{i}(t)
$$

Based on the observed average voltage of each DG, the voltage can be restored in a distributed way as:

$$
u_{i}^{V}(t)=k_{i}^{V P}\left[V^{r e f}-\bar{V}_{i}(t)\right]+k_{i}^{V I} \int\left[V^{r e f}-\bar{V}_{i}(t)\right] d t
$$


where $k_{i}^{V I}, k_{i}^{V P}>0$ and $t \in\left\{t_{k}^{Q i} \mid k=1,2, \ldots\right\}$. The stability analysis is discussed in [32].

\section{HARDWARE-IN-THE-LOOP IMPLEMENTATION}

The proposed controllers in Section III are to be implemented on a cyber-physical MG platform. The details of this platform are introduced as follows:

1) Physical System by OPAL-RT: The electrical network of MGs and all primary controllers are viewed as the physical system and developed in RT-LAB version 11.2. In each control iteration, the data packet of local measurement $\left\{K_{i}^{P} P_{i}, K_{i}^{Q} Q_{i}, \omega_{i}, V_{i}\right\}$ from primary control will be sent to the corresponding R-Pi via user datagram protocol (UDP). The control inputs of $\left\{u_{i}^{P}, u_{i}^{Q}, u_{i}^{\omega}, u_{i}^{V}\right\}$ will be sent back the OPAL-

RT to calculate set points $\omega_{i}^{\text {nom }}$ and $V_{i}^{\text {nom }}$ by (8) and (9).

2) Cyber System by Raspberry Pi: A multi-agent system and its communication network, as the cyber system, are realized by multiple R-Pis. The proposed control are implemented independently on each agent. The Raspberry Pi 3 Model B+ is applied in the HIL experiment and the communications are realised by a network switch.

Each agent or R-Pi is installed with gRPC and has the structure illustrated in Fig. 5. There are three asynchronous processes working concurrently on each agent. In the first process, each agent receives local measurement data from OPAL-RT. In the second process, each agent processes the proposed control algorithm. When the ETC is satisfied, the states $\left\{K_{i}^{P} P_{i}, K_{i}^{Q} Q_{i}, \omega_{i}, V_{i}\right\}$ will be sent to neighbours using gRPC server/client structure over the TCP/IP protocol, and the control signals $\left\{u_{i}^{P}, u_{i}^{Q}, u_{i}^{\omega}, u_{i}^{V}\right\}$ will be updated. The third process sends the control signals from each agent to the corresponding local controller in OPAL-RT.

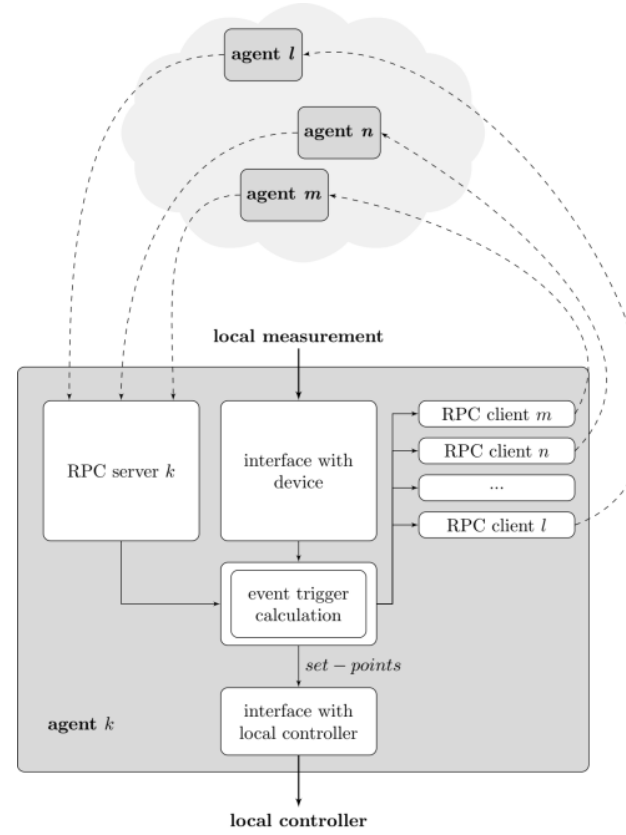

Fig. 5. The structure of an agent implementing the proposed event-triggered algorithm.

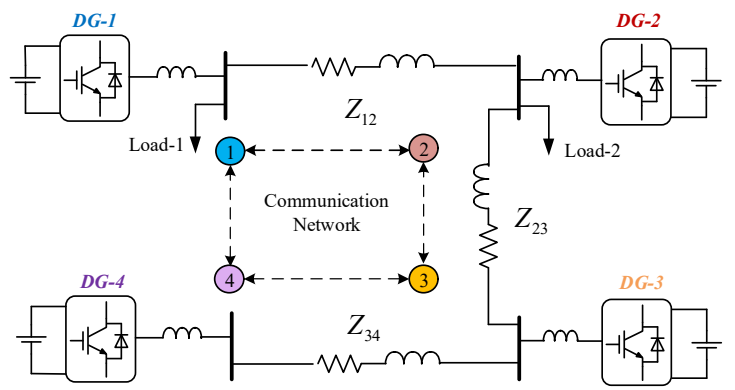

Fig. 6. Single line diagram of the 4-DG MG.

\section{EXPERIMENTAL TEST RESUlts}

The proposed control scheme is firstly tested on a 4-DG MG on the developed platform in Case 1 and Case 2, and then tested on a larger MG system with 13 buses in Case3.

The single line diagram of the 4-DG MG and its associated communication topology is shown in Fig. 6. The parameters of the test MG and the secondary controllers are given in Table I and Table II, respectively. As an example, the adjacent matrix, in-degree matrix and Laplacian matrix of the communication topology in Fig. 6 are shown below.

$$
\begin{gathered}
\mathcal{A}=\left[\begin{array}{llll}
0 & 1 & 0 & 1 \\
1 & 0 & 1 & 0 \\
0 & 1 & 0 & 1 \\
1 & 0 & 1 & 0
\end{array}\right], \mathcal{D}=\left[\begin{array}{llll}
2 & 0 & 0 & 0 \\
0 & 2 & 0 & 0 \\
0 & 0 & 2 & 0 \\
0 & 0 & 0 & 2
\end{array}\right] \\
\mathcal{L}=\left[\begin{array}{cccc}
2 & -1 & 0 & -1 \\
-1 & 2 & -1 & 0 \\
0 & -1 & 2 & -1 \\
-1 & 0 & -1 & 2
\end{array}\right] .
\end{gathered}
$$

Fig. 7 shows the HIL experiment setup in Nanyang Technological University, Singapore. The platform has four RPis, OPAL-RT and server desktop, with their data exchange via a 16-port gigabit switch. Each R-Pi corresponds a secondary controller of a DG, which runs the proposed algorithm. The server desktop works to monitor and manage the R-Pi cluster and OPAL-RT.

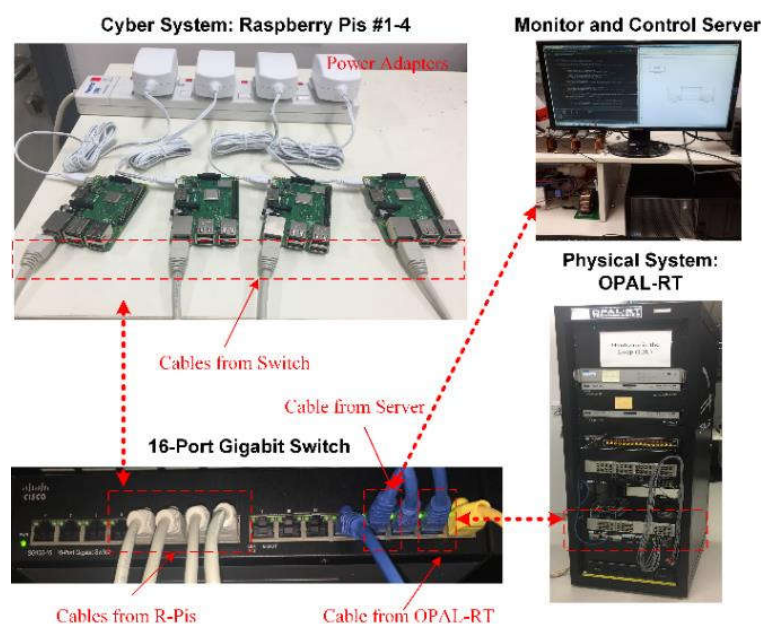

Fig. 7. HIL experiment platform with the four R-Pis. 
TABLE I

PARAmeter Settings OF THE MG Testbed

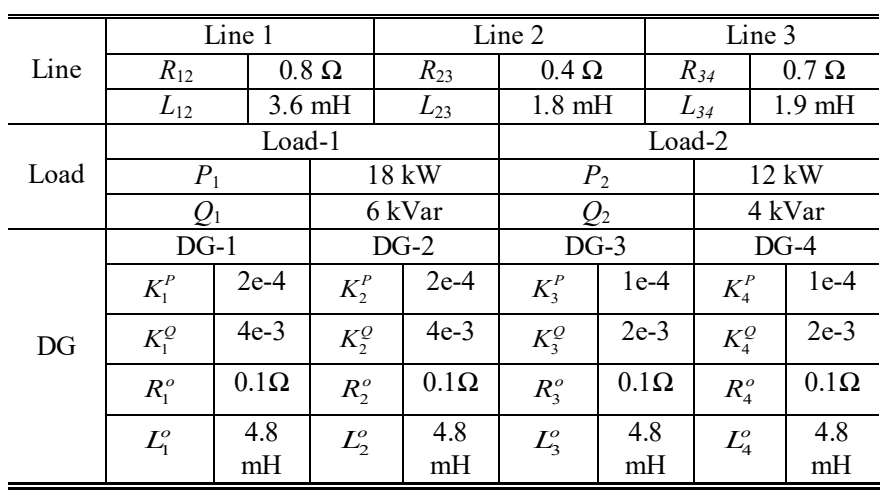

TABLE II

PARAMETER SETTINGS OF THE DistRIBUTED CONTROLLERS

\begin{tabular}{c|c}
\hline \hline $\begin{array}{c}\text { Real Power } \\
\text { Controller }\end{array}$ & $k_{1}^{P}=k_{2}^{P}=k_{3}^{P}=k_{4}^{P}=1$ \\
\hline ETC\#1 & $\sigma_{1}^{P}=\sigma_{2}^{P}=\sigma_{1}^{P}=\sigma_{1}^{P}=0.5$ \\
& $\alpha_{1}^{P}=\alpha_{2}^{P}=\alpha_{3}^{P}=\alpha_{4}^{P}=0.2$ \\
\hline $\begin{array}{c}\text { Frequency } \\
\text { Controller }\end{array}$ & $k_{1}^{\omega}=k_{2}^{\omega}=k_{3}^{\omega}=k_{4}^{\omega}=20$ \\
\hline $\begin{array}{c}\text { Reactive Power } \\
\text { Controller }\end{array}$ & $k_{1}^{Q}=k_{2}^{Q}=k_{3}^{Q}=k_{4}^{Q}=1$ \\
\hline ETC\#2 & $\sigma_{1}^{Q}=\sigma_{2}^{Q}=\sigma_{1}^{Q}=\sigma_{1}^{Q}=0.5$ \\
& $\alpha_{1}^{Q}=\alpha_{2}^{Q}=\alpha_{3}^{Q}=\alpha_{4}^{Q}=0.2$ \\
\hline Voltage & $k_{1}^{V P}=k_{2}^{V P}=k_{3}^{V P}=k_{4}^{V P}=10$ \\
Controller & $k_{1}^{V I}=k_{2}^{V I}=k_{3}^{V I}=k_{4}^{V I}=50$ \\
\hline Reference & $\omega^{\text {ref }}=50 \mathrm{~Hz}, V^{\text {ref }}=230 \sqrt{2} \mathrm{~V}$ \\
\hline \hline
\end{tabular}

\section{A. Case 1: Step Response}

In Case 1, the performance of the proposed controller is validated with step load changes. In the beginning, DGs 1-4 and Load-1 are connected to the MG. Load-2 is connected into the MG at 20s, while Load-1 is switched off from the MG at 40s. The HIL experimental results of real power output and frequency are shown in Figs. 8 and 9. It is observed that the real power is shared inversely proportional to the droop coefficients, as $\left(P_{1}: P_{2}: P_{3}: P_{4}=1: 1: 2: 2\right)$. The frequency can be restored to reference value $(50 \mathrm{~Hz})$ after each load change. The frequency restoration $(\mathrm{t} \approx 0.4 \mathrm{~s})$ responds much faster than the real power sharing $(t \approx 4 s)$ process, which verifies the effectiveness of the timescale decoupling. The HIL experimental results of reactive power output and voltage magnitude are shown in Figs. 10 and 11. The reactive power is also shared inversely proportional to the droop coefficients as $\left(Q_{1}: Q_{2}: Q_{3}: Q_{4}=1: 1: 2: 2\right)$. The estimated average bus voltages can be restored to the reference value $(230 \sqrt{2} \mathrm{~V})$ as shown in Fig. 11 (b), and the actual bus voltages are also restored to some extent in Fig. 11 (a). The voltage restoration $(t \approx 1.4 \mathrm{~s})$ also operates much faster than reactive power sharing $(\tau \approx 14 \mathrm{~s})$.

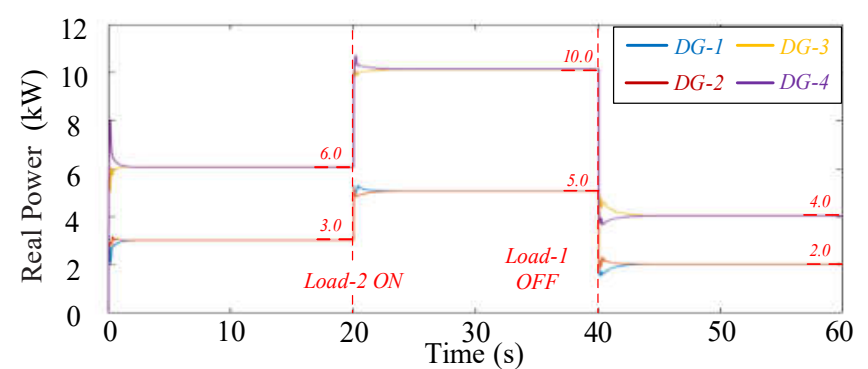

Fig. 8. The real power output of each DG in Case 1.

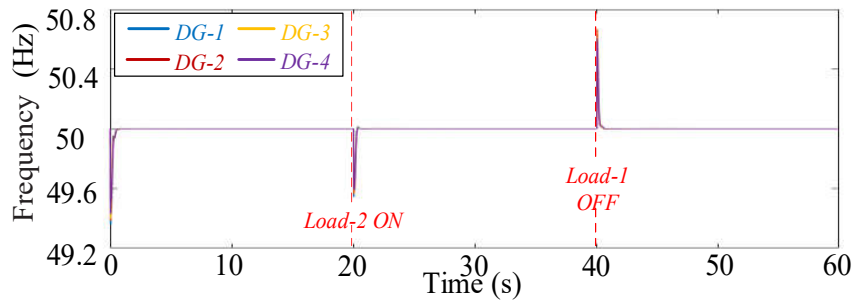

Fig. 9. The frequency of each DG in Case 1.

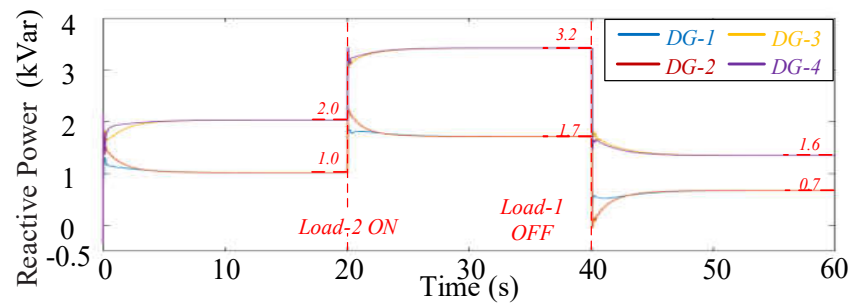

Fig. 10. The reactive power of each DG in Case 1.

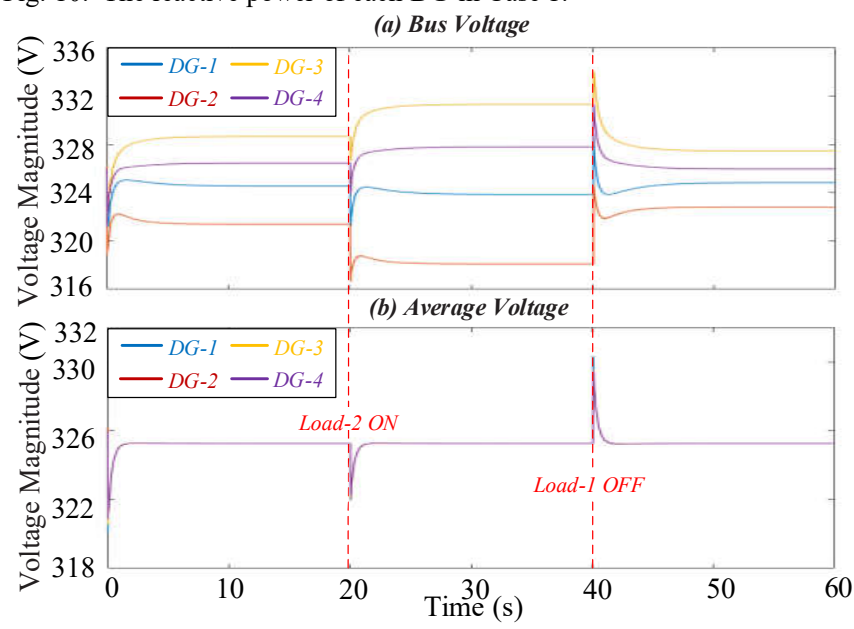

Fig. 11. The voltage magnitude of each DG in Case 1.

\section{B. Effectiveness of the Event-Triggered Control}

The effectiveness of the proposed event-triggered control is discussed in this subsection. The event-triggered time sequence of each agent (DG) in Case 1 is shown in Figs. 12 and 13. The secondary control on each agent is triggered asynchronously according to its own ETCs. Here we take Agent-1 as an example. In Fig. 12, Agent-1 is triggered by ETC\#1 last for 4s. In Fig. 13 Agent- 1 is triggered by ETC\#2 last for $14 \mathrm{~s}$. The total communications of Agent-1 are governed by two ETCs together. As compared to conventional control with continuous communications in Figs. 12 and 13, the proposed method can significantly reduce communications, both during the event and after the event. In the meantime, the details of the total eventtriggered time for Agent-1 during 20s to 40s are shown in Fig. 14. It can be observed that the frequency of communication is 
significantly reduced. The data flows are turned to idle when the system comes into the steady-state.

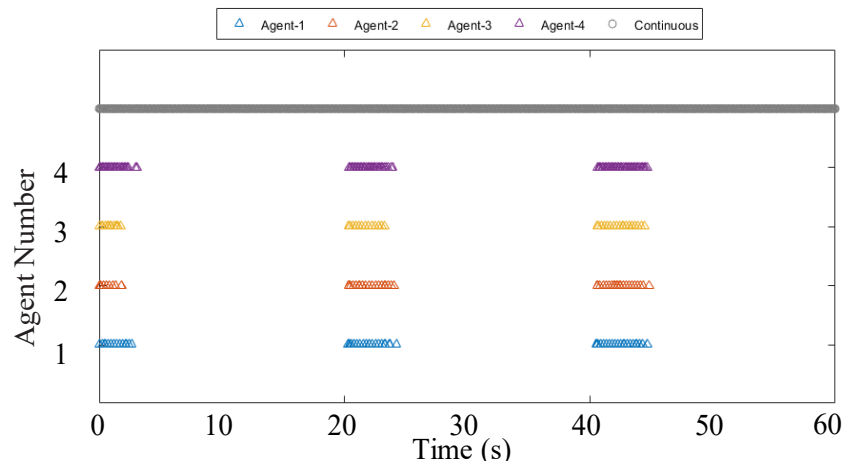

Fig. 12. The event-triggered time of each agent (DG) under ETC-1 (Continuous: conventional method).

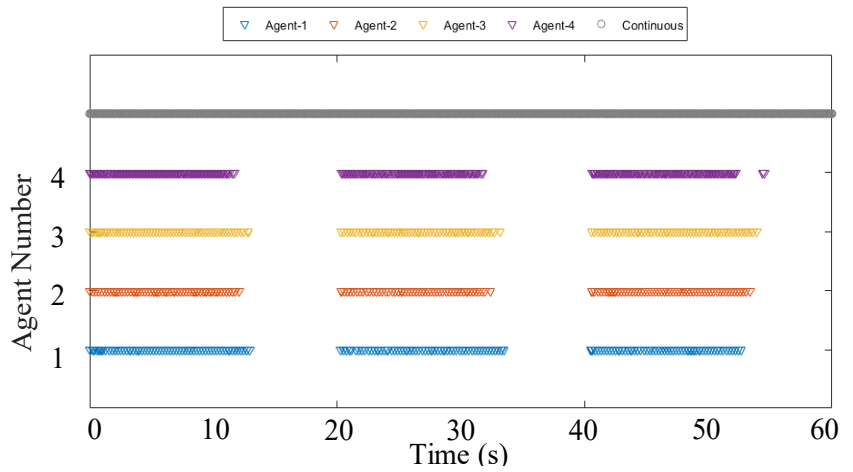

Fig. 13. The event-triggered time of each agent (DG) under ETC-2. (Continuous: conventional method).

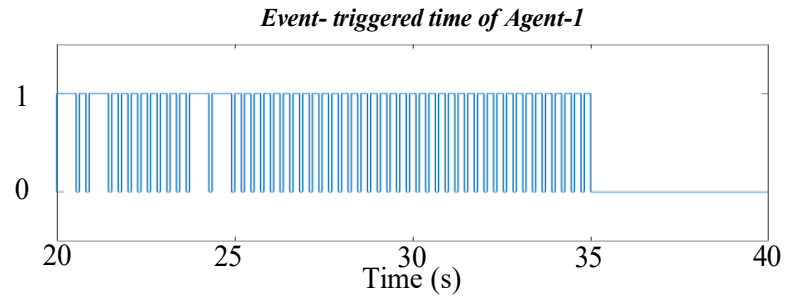

Fig. 14. The total event-triggered time of Agent-1. (1: Triggered. 0-Not triggered).

\section{Case 2: Communication Failures and Topology Change}

In Case 2, the performance of the proposed method is validated under communication failures and topology change. The physical and cyber events in Case 2 is shown in Fig. 15. In the physical system, there are loads switched on and off events. Load-2 is switch into the MG at 20s. Load-1 is disconnected from $\mathrm{MG}$ at $40 \mathrm{~s}$, and then reconnected at $60 \mathrm{~s}$. In the cyber system, the topologies are changed to emulate communication failures. The cyber link 1-2 fails at $15 \mathrm{~s}$, followed by the loss of cyber link 1-4 at 35s. Afterward, the cyber link 1-2 is restored at $55 \mathrm{~s}$. The list of neighbours of each agent (DG) is configured initially corresponding to the communication topology in Fig. 6 (Agent-1: [2, 4], Agent-2: [1, 3], Agent-3 [2, 4], Agent-4: [1, 3]). Each agent is able to adapt the alteration of the network topology by online self-adjusting the list of neighbour. For example, the communication of each DG is reconfigured as (Agent-1: [4], Agent-2: [3], Agent-3: [2, 4], Agent-4: [1,3]) at $15 \mathrm{~s}$.
The HIL experimental results of real power, frequency, reactive power and bus voltage of each DG are shown in Figs. $16-19$, respectively. It can be found that the system converges slower $(15 \mathrm{~s}-100 \mathrm{~s})$ compared to the original graph $(0-15 \mathrm{~s})$ due to the loss of cyber links. The proportional power sharing is ensured as long as the communication graph is still completed. From $35-55 \mathrm{~s}$, the DG-1 is isolated without any cyber links thus it is controlled by only droop control. As a result, the proportional real and reactive power sharing cannot be maintained during this period as shown in Figs. 16 and 18.

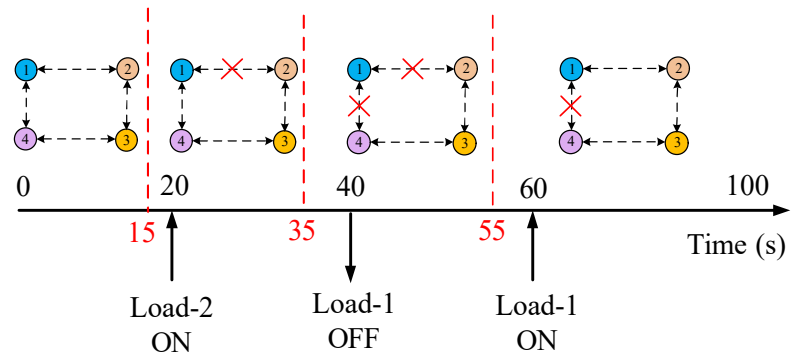

Fig. 15. Cyber and physical events of Case 2.

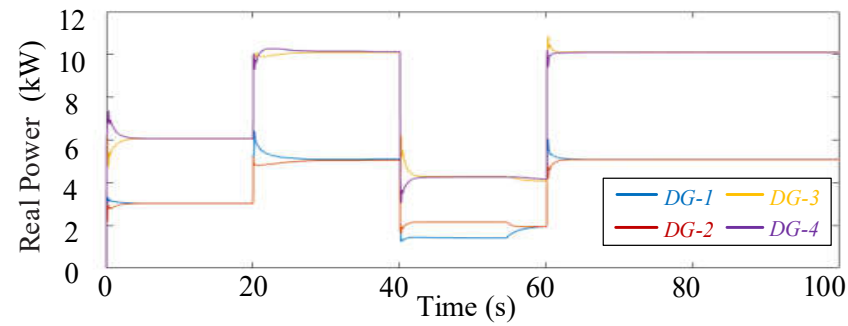

Fig. 16. The real power output of each DG in Case 2.

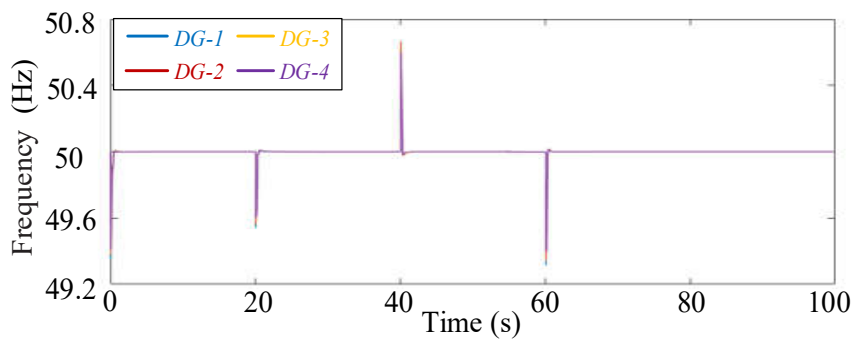

Fig. 17. The frequency of each DG in Case 2.

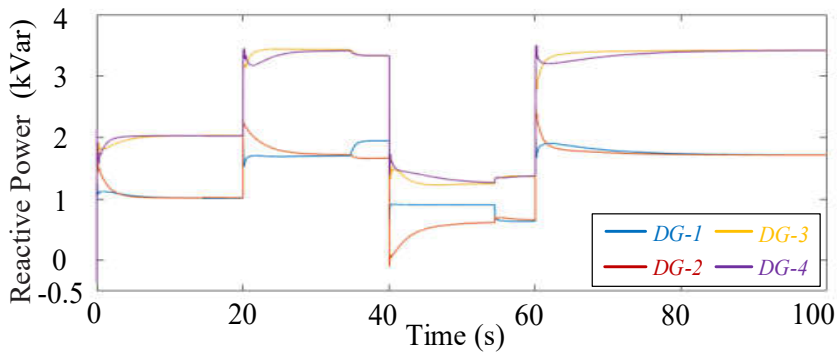

Fig. 18. The reactive power of each DG in Case 2.

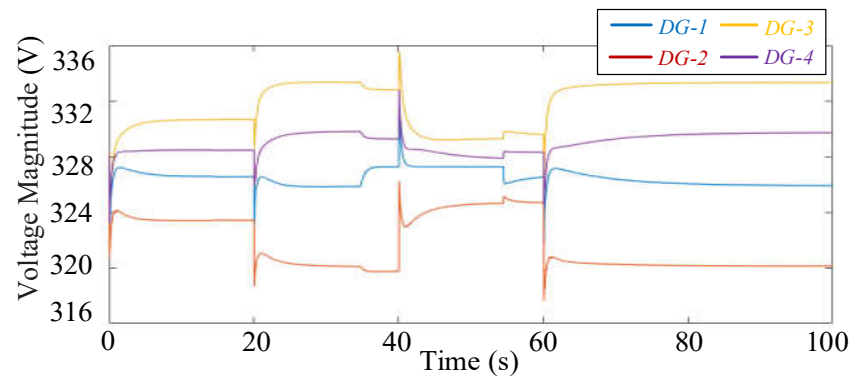

Fig. 19. The voltage magnitude of each DG in Case 2. 


\section{Case 3: Scalability Test}

In Case 3, the performance of the proposed method is further validated in a larger system with realistic PV and load profiles. The electrical and communication topology of the tested MG is shown in Fig. 20, which is modified from the IEEE 13-bus system [33]. The droop control coefficients of DGs 1-6 are considered with the ratio of $4: 4: 2: 2: 1: 1$. The PV and load profiles considered in this case are given in Fig. 21 .

The OPAL-RT test results of real power, frequency, reactive power, bus voltage and estimated average voltage for each DG are shown in Figs. 22-26, respectively. It can be found that, regardless of $\mathrm{PV}$ and load variations, the real and reactive power can be shared reversely proportional to the droop coefficients as 1:1:2:2:4:4 for DGs 1-6 in Fig. 22 and 24. The frequency and estimated average voltage can be regulated around the reference values in Fig 23 and 26. In addition, the event-triggered time of each agent during $100 \mathrm{~s}$ to $105 \mathrm{~s}$ are shown in Fig. 27. It can be observed that the frequency of communication is reduced by the proposed approach.

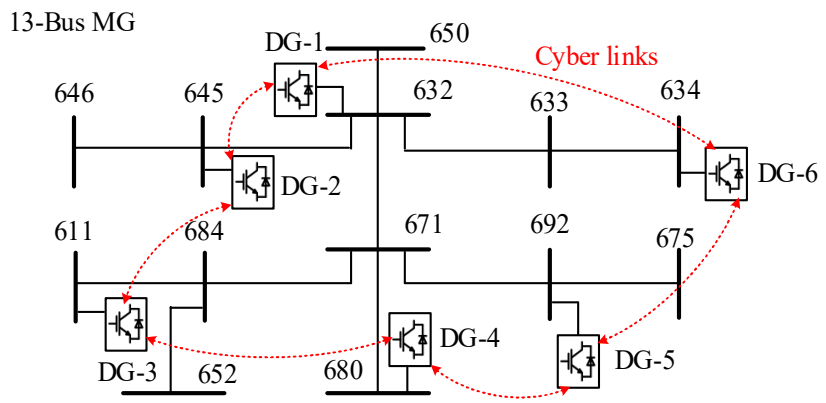

Fig. 20. Electrical and communication topology of the 13-bus MG in Case 3.

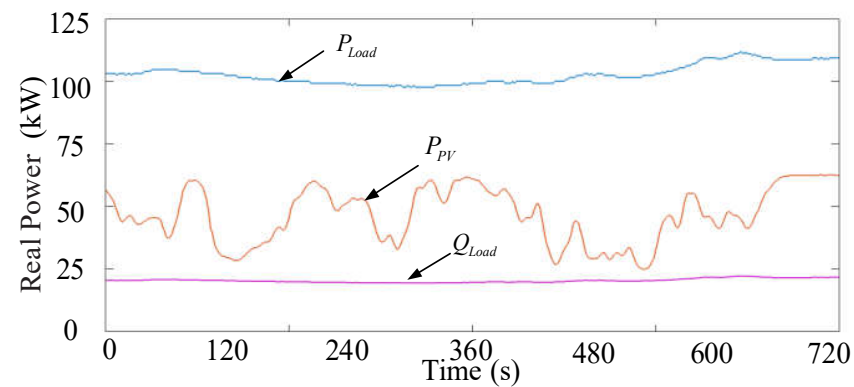

Fig. 21. The PV and load profiles used in Case 3.

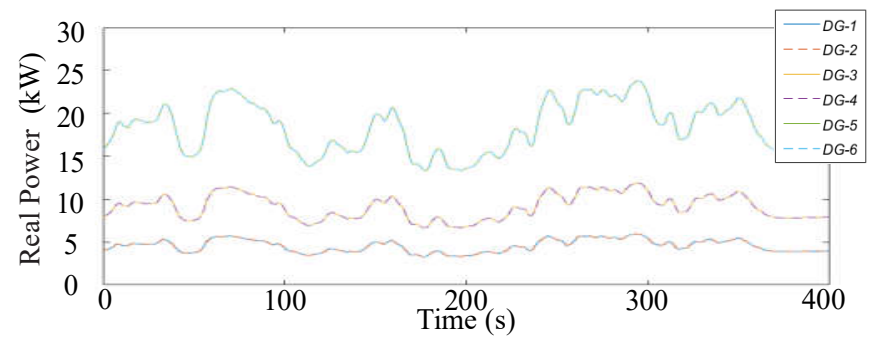

Fig. 22. The real power output of each DG in Case 3.

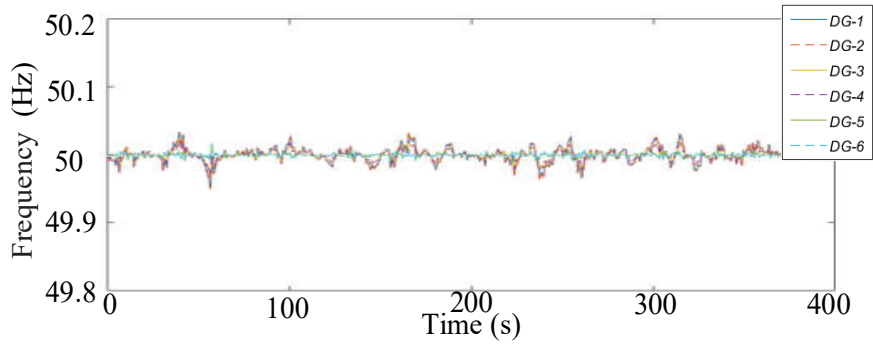

Fig. 23. The frequency of each DG in Case 3.

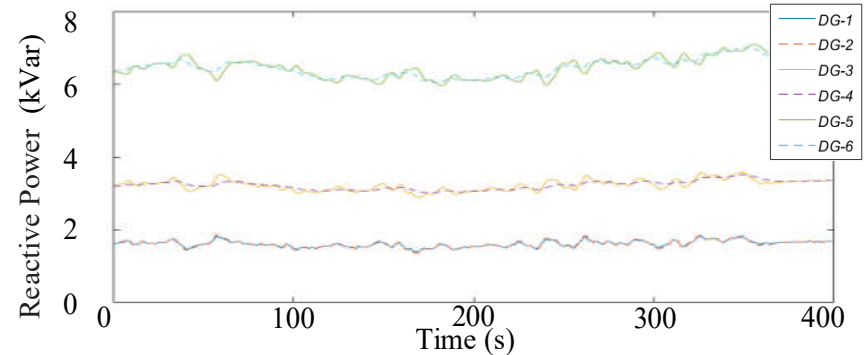

Fig. 24. The reactive power of each DG in Case 3 .

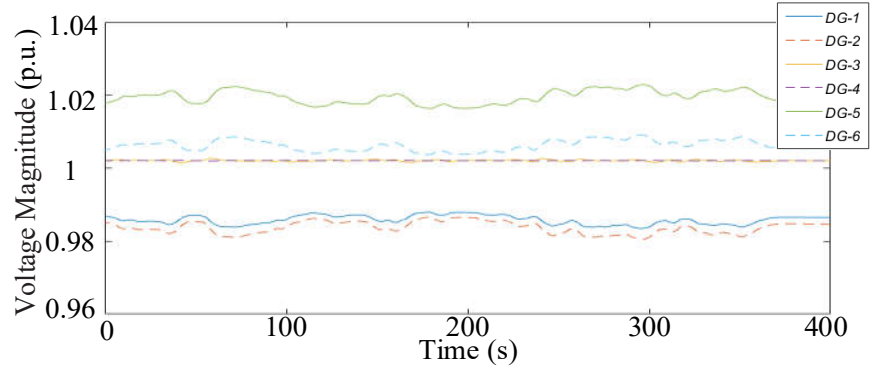

Fig. 25. The voltage magnitude of each DG in Case 3.

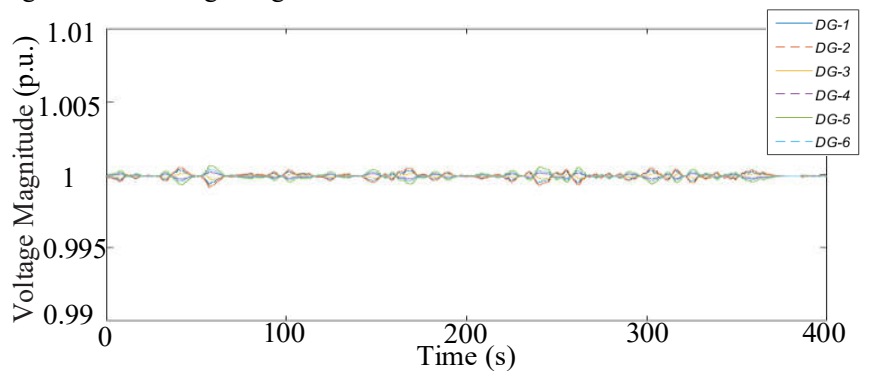

Fig. 26. The estimated average voltage of each DG in Case 3.

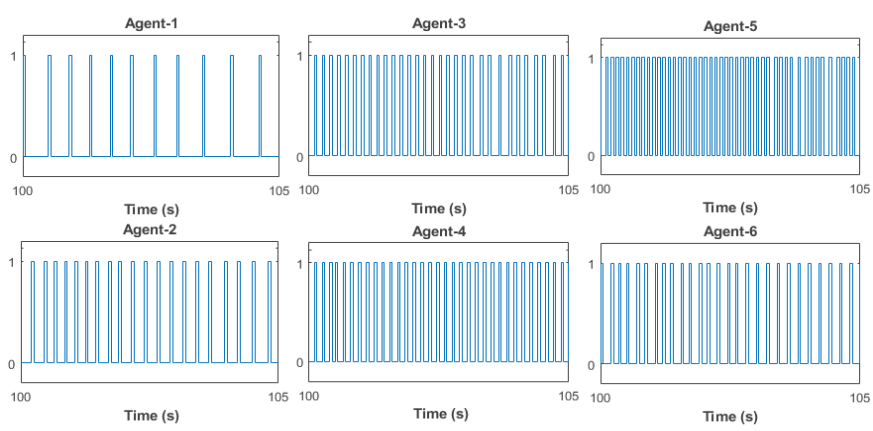

Fig. 27. The event-triggered time of each agent (DG) in Case 3. (1: Triggered. 0-Not triggered).

\section{CONCLUSION}

In this paper, the distributed event-triggered secondary control for islanded MGs is proposed with the cyber-physical design and implementation. The secondary controllers are decoupled into two timescales to facilitate the proposed distributed controllers and ETCs. The theoretical analysis and 
closely practical implementation of the proposed method are discussed.

The proposed secondary controllers have been built and tested on the cyber-physical MG platform. The HIL experimental results validate the effectiveness of the proposed method. The advantages of the proposed approach over periodical communication methods are demonstrated. The control performance under step load changes and communication failures is evaluated. Besides, the proposed method is also applied for a larger scale system with realistic profiles. Thus this paper provides a practical design and implementation guidance of communication efficient secondary control of MGs.

\section{REFERENCES}

[1] R. H. Lasseter, J. H. Eto, B. Schenkman, J. Stevens, et al., "CERTS MG laboratory testbed," IEEE Trans. Power Delivery, vol. 26, no. 1, pp. 325332, Jan. 2011.

[2] D. E. Olivares, et al. "Trends in MG control," IEEE Trans. Smart Grid, vol. 5, no. 4, pp. 1905-1919, Jul. 2014.

[3] I. U. Nutkani, P. C. Loh, and F. Blaabjerg, "Distributed operation of interlinked AC microgrids with dynamic active and reactive power tuning," IEEE Trans. Industry Applications, vol. 49, no .5, pp. 2188-2196, 2013.

[4] A. Nelson, et al. "Cyber-physical test platform for MGs: Combining hardware, hardware-in-the-loop, and network-simulator-in-the-loop," Power and Energy Society General Meeting (PESGM), 2016.

[5] X. Ge, F. Yang, Q. Han, "Distributed networked control systems: A brief overview," Information Sciences. vol. 380, pp. 117-31, Feb. 2017.

[6] F. Dörfler, J. W. Simpson-Porco, and F. Bullo. "Breaking the hierarchy: Distributed control and economic optimality in microgrids," IEEE Transactions on Control of Network Systems, vol. 3, no .3, pp. 241-253, 2015.

[7] C. Deng and G. H. Yang, "Distributed adaptive fault-tolerant control approach to cooperative output regulation for linear multi-agent systems," Automatica, vol. 103, pp. 62-68, May 2019.

[8] J. M. Guerrero, J. C. Vasquez, J. Matas, and L. G. de Vicuna, "Hierarchical control of droop-controlled ac and dc MGs-A general approach toward standardization," IEEE Trans. Ind. Electron., vol. 58, no. 1, pp. 158-172, Jan. 2011.

[9] A. Bidram, and A. Davoudi. "Hierarchical structure of MGs control system," IEEE Trans. Smart Grid, vol. 3, no .4, pp. 1963-1976, 2012.

[10] J. P. Lopes, C. L. Moreira, and A. G. Madureira. "Defining control strategies for MGs islanded operation," IEEE Trans. Power Systems vol. 21, no .2, pp. 916-924, 2006.

[11] F. Guo, et al. "Distributed secondary voltage and frequency restoration control of droop-controlled inverter-based MGs," IEEE Trans. Industrial Electronics, vol. 62, no .7, pp. 4355-4364, Jul. 2015.

[12] J. Zhou, et al. "Consensus-based Distributed Control for Accurate Reactive, Harmonic and Imbalance Power Sharing in MGs," IEEE Trans. Smart Grid, vol. 9, no. 4, pp. 2453-2467, Jul. 2018.

[13] R. Han, et al. "Containment and consensus-based distributed coordination control to achieve bounded voltage and precise reactive power sharing in islanded AC MGs," IEEE Trans. Industry Applications, vol. 53, no .6, pp. 5187-5199, Nov. 2017.

[14] C. Zhang, et al. "Robustly coordinated operation of a multi-energy microgrid with flexible electric and thermal loads," IEEE Trans. Smart Grid, 2018.

[15] Y. Wang, et al. "Aggregated energy storage for power system frequency control: a finite-time consensus approach," IEEE Trans. Smart Grid, 2018

[16] A. Kaur, J. Kaushal, and P. Basak, "A review on MG central controller," Renewable and Sustainable Energy Reviews, vol. 55, pp. 338-345, Mar. 2016.

[17] J. Simpson-Porco, et al. "Secondary frequency and voltage control of islanded MGs via distributed averaging," IEEE Trans. Industrial Electronics, vol. 62, no .11, pp. 7025-7038, Nov. 2015.

[18] Y. Wang, et al. "Distributed Optimal Control of Reactive Power and Voltage in Islanded MGs," IEEE Trans. Industry Applications, vol. 53, no. 1, pp. 340-349, Jan.-Feb. 2017.

[19] R. Zhang, and B. Hredzak. "Distributed Finite-Time Multi-Agent Control for DC MGs with Time Delays," IEEE Trans. Smart Grid, 2018.
[20] D. V. Dimarogonas, E. Frazzoli, K. H. Johansson, "Distributed eventtriggered control for multi-agent systems," IEEE Trans. Automatic Control, vol. 57, no. 5, pp. 1291-1297, May 2012.

[21] M. Zhao, C. Peng, W. He, and Y. Song, "Event-Triggered Communication for Leader-Following Consensus of Second-Order Multiagent Systems," IEEE Trans. Cybernetics, vol. 48, no. 6, pp. 1888 1897, 2018.

[22] S. Weng, D. Yue, S. Member, C. Dou, J. Shi, and C. Huang, "Distributed Event-Triggered Cooperative Control for Frequency and Voltage Stability and Power Sharing in Isolated Inverter-Based MG," IEEE Trans. Cybernetics, 2018.

[23] Y. Fan, G. Hu, and M. Egerstedt, "Distributed Reactive Power Sharing Control for MGs with Event-Triggered Communication," IEEE Trans. Control Syst. Technol., vol. 25, no. 1, pp. 118-128, 2017.

[24] M. Chen, X. Xiao, and J. M. Guerrero, "Secondary Restoration Control of Islanded MGs With Decentralized Event-triggered Strategy," IEEE Trans. Ind. Informatics, 2017.

[25] C. W. Zhao, J. Jegatheesan, and S. C. Loon, "Exploring IoT application using raspberry pi," International Journal of Computer Networks and Applications, vol. 2, no .1, pp. 27-34, Jan. 2015.

[26] T. Nguyen, et al. "Multi-agent system with plug and play feature for distributed secondary control in microgrid - Controller and power hardware-in-the-loop Implementation," Energies, vol. 11, no .12, pp. 3253,2018

[27] Y. Wang, et al. "Distributed Event-Triggered Control for Islanded Microgrids: Cyber-Physical Design and Implementation," 2019 IEEE/IAS 55th Industrial and Commercial Power Systems Technical Conference (I\&CPS). IEEE, 2019.

[28] P. Lin, et al. "A Distributed Power Management Strategy for MultiParalleled Bidirectional Interlinking Converters in Hybrid AC/DC Microgrids," IEEE Trans. Smart Grid, 2019.

[29] Y. Qi, et al. "Two-Dimensional Impedance-Shaping Control with Enhanced Harmonic Power Sharing for Inverter-Based Microgrids," IEEE Trans. Power Electronics, 2019.

[30] F. L. Lewis, H. Zhang, K. Hengster-Movric, and A. Das, Cooperative control of multi-agent systems: optimal and adaptive design approaches. Springer Science \& Business Media, 2013.

[31] X. Ge, and Q. Han. "Distributed event-triggered $\mathrm{H} \infty$ filtering over sensor networks with communication delays," Information Sciences, vol. 291, pp. 128-142, Jan. 2015.

[32] A. Bidram, V. Nasirian, A. Davoudi, and F. L. Lewis, "Droop-free distributed control of AC MGs," IEEE Trans. Power Electronics, vol. 31, no. 2, pp. 1600-1671, Feb. 2016.

[33] T. Morstyn, B. Hredzak, and V. G. Agelidis. "Distributed cooperative control of microgrid storage," IEEE Trans. Power Systems, vol. 30, no .5 pp. 2780-2789, Oct. 2014.

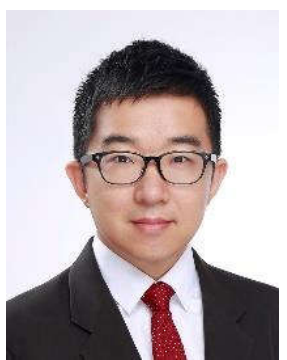

Yu Wang (S'12-M'17) received the B.Eng. degree from Wuhan University, Wuhan China in 2011, and the M.Sc. and Ph.D. degree from Nanyang Technological University, Singapore in 2012 and 2017 , respectively. He is currently a research fellow in Nanyang Technological University, Singapore. His research interests include distributed control and optimization, grid energy storage systems, renewable energy integration, microgrids and smart grids.

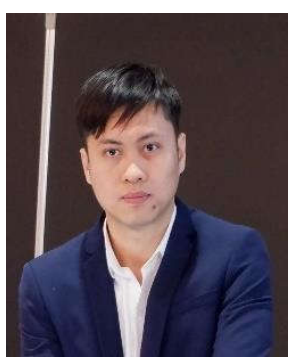

Tung-Lam Nguyen received the Master's degree in electrical engineering from National Taiwan University of Science and Technology, Taiwan, in 2014, and the Ph.D. degree from the University of Grenoble Alpes, Grenoble, France, in 2019. He is currently a Lecturer with the Faculty of Electrical Engineering, The University of Danang, University of Science and Technology, Danang, Vietnam, and a Post-Doctoral Researcher with the Grenoble Institute of Technology, Grenoble, France, as part of the G2ELab. His research interests include distributed control and optimization in microgrids and distribution networks, and co-simulation in smart grid. 

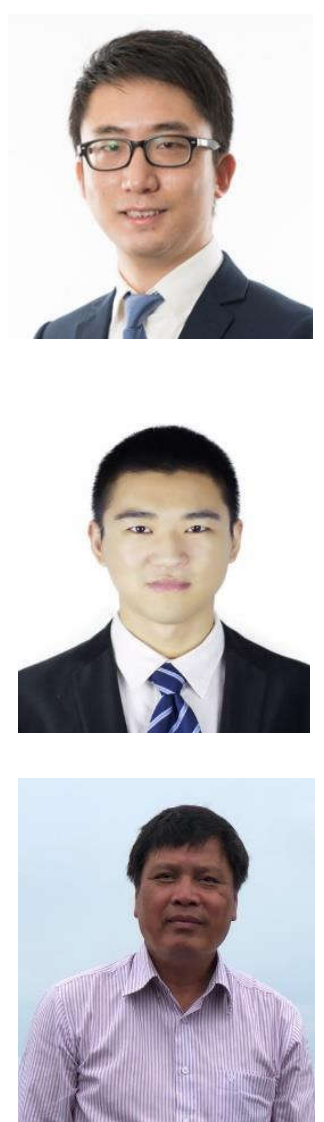

Quoc-Tuan Tran received his Ph.D. degree in Electrical Engineering and Habilitation à Diriger des Recherches degree from the Grenoble Institute of Technology in 1993 and 2000, respectively. He is an IEEE senior member. He is currently a Professor with INSTN - Paris Saclay University, and a Scientific Manager with Alternative Energies and Atomic Energy Commission (CEA) - National Institute for Solar Energy (INES). His research interests are in the fields of power system analysis, operations, electromagnetic transients, distributed generation, smart-grid and renewable energy.

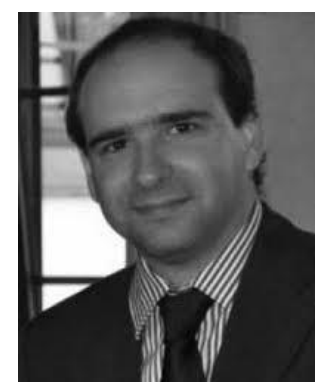

Raphael Caire received the Diplôme d'Etudes Approfondies and Doctorat de l'INPG degrees from the Institut National Polytechnique de Grenoble in 2000 and 2004, respectively. He has been with the Power Electronics field at the Center of Power Electronic System (CPES), USA, since 2000 and within several EDF research centers in Germany and France from 2004 to 2006 $\mathrm{He}$ is currently an Associate Professor with the Grenoble Institute of Technology, Ecole d'ingénieurs en énergie eau et environnement, Grenoble Electrical Engineering Laboratory. His research is centered on the impacts, production control of dispersed generation on distribution system and critical infrastructures. 